\title{
Ocorrência de fungos em sementes de plantas medicinais, aromáticas e condimentares da família Lamiaceae
}

\author{
Pedro C. Kruppa \& Olga M.R. Russomanno \\ Centro de Pesquisa e Desenvolvimento de Sanidade Vegetal, Instituto Biológico, 04014-002, São Paulo, SP, Brasil.
}

Autor para correspondência: Pedro C. Kruppa, e-mail: pckruppa@biologico.sp.gov.br

\begin{abstract}
RESUMO
Tendo em vista a importância das sementes como portadoras de fungos que transmitem doenças às plantas, foram avaliadas 117 amostras de sementes da família Lamiaceae. Amostras de alecrim, lavanda, erva-cidreira, hissopo, hortelã-pimenta, manjericão, manjerona, orégano, sálvia, segurelha e tomilho foram analisadas pelo método do papel de filtro. Foram encontrados fungos em $71,8 \%$ das amostras avaliadas, envolvendo 24 gêneros, entre eles, Alternaria, Bipolaris, Colletotrichum, Exserohilum, Fusarium e Phoma. Entre as espécies fitopatogênicas importantes foram identificados Fusarium oxysporum em manjericão, F. solani em manjericão e orégano, Colletotrichum gloeosporioides em hissopo, hortelã-pimenta e manjericão e Bipolaris sorokiniana em manjericão e tomilho. Considerando que $47,9 \%$ das amostras analisadas foram tratadas com o fungicida captam, é possível concluir que a qualidade sanitária das sementes avaliadas não é satisfatória.
\end{abstract}

Palavras-chave: patologia de sementes, sanidade, fitopatógenos, ascomicetos, diagnose.

\begin{abstract}
Occurrence of fungi in medicinal, aromatic and spice plant seeds of Lamiaceae

In view of the importance of seeds as carriers of fungi that may transmit diseases to plants, 117 seed samples of the family Lamiaceae were evaluated. Rosemary, lavender, lemon balm, hyssop, basil, marjoram, peppermint, oregano, sage, summer savory and thyme samples were analyzed using blotter test. In these analyses, fungi were detected in $71.8 \%$ of the samples, involving the 24 genera, including Alternaria, Bipolaris, Colletotrichum, Exserohilum Fusarium and Phoma. Among the important plant pathogens, Fusarium oxysporum in basil, F. solani in basil and oregano, Colletotrichum gloeosporioides in hyssop, basil and peppermint and Bipolaris sorokiniana in thyme and basil were detected. Considering that $47.9 \%$ of the analyzed samples were treated with captan fungicide, it can be concluded that the sanitary quality of the evaluated seed samples is not satisfactory.
\end{abstract}

Keywords: seed pathology, seed health, plant pathogens, ascomycetes, diagnosis.

Várias espécies de plantas da família Lamiaceae (=Labiatae) têm importância hortícola e são utilizadas na culinária, na medicina caseira, na indústria farmacêutica e cosmética. Essa família apresenta distribuição cosmopolita, com cerca de 300 gêneros e aproximadamente 7500 espécies. No Brasil, ocorrem cerca de 350 espécies distribuídas em 26 gêneros (Souza, 2005). Muitas espécies de importância econômica da família Lamiaceae, como o alecrim (Rosmarinus officinalis L.), erva-cidreira (Melissa officinalis L.), hissopo (Hyssopus officinalis L.), hortelãpimenta (Mentha piperita L.), lavanda (Lavandula spp.), manjericão (Ocimum basilicum L.), manjerona (Origanum majorana L.), orégano (Origanum vulgare L.), sálvia (Salvia officinalis L.), segurelha (Satureja hortensis L.) e tomilho (Thymus vulgaris L.) são propagadas por sementes.

As doenças fúngicas mais freqüentes nas plantas medicinais, aromáticas e condimentares são: antracnose, ferrugem, carvão, podridão, oídio, míldio e murcha (Correa Jr. et al., 1994). Entretanto, os registros de ocorrência no Brasil de fungos causando doenças em plantas da família Lamiaceae são poucos em relação à quantidade de espécies cultivadas. Há relatos de fungos patogênicos transmitidos por sementes, prejudicando o desenvolvimento de plântulas, causando manchas foliares ou murchas (Urben et al., 1987; Mendes et al., 1998; 2003; Kruppa \& Russomanno, 2001; Russomanno et al., 2004a).

A presença de fungos em sementes, além de possibilitar a introdução de patógenos em áreas de cultivo e ocasionar doenças às culturas agrícolas, pode também provocar alterações no metabolismo das plantas comprometendo suas propriedades terapêuticas e sabor. No armazenamento, alguns fungos provocam alterações físico-químicas nos tecidos das sementes, causando perda de lipídeos, carboidratos, proteínas e aumento de ácido graxo, além de influir na germinação das sementes (Neergaard, 1979; Regina \& Tulasi, 1992). Considerando que a boa qualidade das sementes é pré-requisito para o sucesso da produção agrícola, esse trabalho teve por finalidade investigar a sanidade de amostras comerciais de sementes de algumas espécies de plantas da família Lamiaceae.

Os testes de sanidade das sementes foram realizados no Laboratório de Patologia de Sementes do Centro de Pesquisa e Desenvolvimento de Sanidade Vegetal do Instituto Biológico 
de São Paulo. Foram coletadas 117 amostras envolvendo cinco marcas comerciais obtidas em diferentes pontos no município de São Paulo SP, no período de 2003 a 2006. A maioria das amostras foi adquirida em embalagens com revestimento de alumínio, à prova de umidade, enquanto que algumas outras não, conforme a marca da empacotadora. As embalagens estavam identificadas com o número do lote, a porcentagem de germinação, o prazo de validade e porcentagem de pureza, porém, apenas uma marca comercial identificava a origem das sementes. No rótulo das embalagens de três marcas constavam que as sementes foram tratadas com o fungicida captam $75 \%$ (na concentração de $0,15 \%$ ou $0,20 \%$ ), enquanto que nas demais não constava qualquer indicação de tratamento fungicida.

Foram analisadas 8 amostras de alecrim, 13 de ervacidreira, 7 de hissopo, 9 de hortelã-pimenta, 5 de lavanda, 7 de manjerona, 28 de manjericão, 11 de orégano, 10 de sálvia, 9 de segurelha e 10 de tomilho pelo método de papel de filtro (Neergaard, 1979). Empregaram-se 200 sementes por amostra, distribuídas em placas de poliestireno de $9 \mathrm{~cm}$ de diâmetro contendo três discos de papel de filtro umedecidos com água destilada. Foram distribuídas 10 ou 25 sementes por placa, conforme o tamanho das sementes. As sementes foram incubadas à temperatura em torno de $20^{\circ} \mathrm{C}$, em ciclo de $12 \mathrm{~h}$ de luz negra (320-400nm) e ausência de luminosidade, por 8 dias. Após o período de incubação, as sementes foram observadas individualmente sob microscópio estereoscópio para a identificação dos fungos. Quando necessário, foram realizadas preparações microscópicas para a identificação das espécies (Malone \& Muskett, 1964; Booth, 1971; Ellis, 1971; Barnett \& Hunter, 1972; Neergaard, 1979; Singh et al., 1991). Os resultados foram avaliados pela freqüência relativa dos fungos presentes nas amostras de sementes, dada pela fórmula $\mathrm{Fr}=(\mathrm{n} / \mathrm{N}) \times 100$, onde $\mathrm{n}=$ número de amostras com fungo e $\mathrm{N}=$ número de amostras por espécie de semente.

As análises de sanidade revelaram a presença de 24 gêneros de fungos, sendo os que apresentaram maior freqüência relativa total Rhizopus, Cladosporium, Alternaria, Aspergillus, Epicoccum, Penicillium, Trichoderma, Fusarium e Mucor (Tabela 1). Espécies dos gêneros Acremoniella, Aristatoma, Aspergillus, Chaetomium, Doratomyces, Epicoccum, Mucor, Penicillium, Pithomyces, Rhizopus, Trichoderma e Ulocladium são consideradas contaminantes em sementes (Malone \& Muskett, 1964; Barnett \& Hunter, 1972). Acremonium (syn. Cephalosporium), Cladosporium, Curvularia, Fusicoccum, Pestalotiopsis e Stemphylium também aparecem como contaminantes em sementes, mas em alguns casos, atuam como patógenos e, dependendo da espécie fúngica e da planta hospedeira, podem ocasionar doenças (Malone \& Muskett, 1964; Richardson \& Noble, 1968). Os gêneros Alternaria, Bipolaris, Colletotrichum, Exserohilum, Fusarium e Phoma possuem espécies que podem causar importantes doenças, conforme o hospedeiro envolvido (Richardson \& Noble, 1968; Neergaard, 1979).

A espécie Fusarium oxysporum Schlecht., detectada na amostra de sementes de manjericão, já foi relatada causando doenças nesse hospedeiro (Garibaldi et al.,
1997). No Brasil, essa espécie foi constatada em plantas de manjericão (Mendes et al., 2003) e manjericão anão ( $O$. minimum L.) (Russomanno et al., 2004 a). F. solani (Mart.) Sacc., detectada nas amostras de sementes de manjericão e orégano foi descrita causando murcha neste último hospedeiro (Garbagnoli \& Gaetan, 1994). Nas sementes de erva-cidreira, manjericão e segurelha foram detectadas algumas espécies de Fusarium que não foram identificadas. Há relatos de Fusarium sp. causando doenças em ervacidreira (Machowicz-Stefaniak et al., 2002; 2004).

Colletotrichum gloeosporioides (Penz.) Sacc., detectada em amostras de sementes de hissopo, manjericão e hortelã-pimenta já foi relatada causando doenças foliares em hortelã-pimenta (Gaetan \& Gally, 1993) e manjericão (Garibaldi et al., 1997). O gênero Phoma presente em amostras de sementes de alecrim, manjericão, sálvia e tomilho, já foi relatado causando doenças em erva-cidreira, (Machowicz-Stefaniak et al., 2002, 2004) e tomilho (Machowicz-Stefaniak et al., 2004).

Alternaria alternata (Fr.) Keissl. (syn. A. tenuis Nees) foi constatada nas amostras de sementes de 9 espécies, com uma freqüência de 22,2 \%. Essa espécie foi relatada causando doenças em alecrim (Perello \& Bello, 1995), erva-cidreira (Machowicz-Stefaniak et al., 2002, 2004), manjericão (Kruppa \& Russomanno, 2001), hortelãzinha (Mendes et al., 1998) e tomilho (Surviliene \& Dambrauskiene, 2006).

Os gêneros Aspergillus e Penicillium, presentes em $18,8 \%$ e $16,2 \%$ das amostras de sementes analisadas, respectivamente, são importantes fungos de armazenamento e a sua presença geralmente indica a má qualidade ou problemas de conservação das sementes (Neergaard, 1979). Esses gêneros, juntamente com Fusarium, presente em $11 \%$ das amostras, também apresentam espécies produtoras de micotoxinas (Aziz et al., 1998; Singh et al., 1991). Russomanno et al. (2004 b) relatam a ocorrência desses fungos em materiais comercializados na forma de chás. Dos fungos presentes nas amostras não foram encontrados relatos no Brasil de espécies de Bipolaris, Colletotrichum, Exserohilum, Fusarium equiseti, $F$. moniliforme, F. solani, F. semitectum e Phoma ocorrendo nas sementes avaliadas.

Em 71,8\% das 117 amostras analisadas foi detectada a presença de fungos (Tabela 2). O captam, fungicida usado pelas empresas sementeiras no tratamento de sementes de 47,9 $\%$ das amostras avaliadas, não evitou a presença de fungos em 35 amostras, correspondendo a 62,5\% das amostras tratadas. Fungos como Alternaria, Aspergillus, Bipolaris, Colletotrichum, Fusarium, Penicillium, Pestalotiopsis e Phoma não foram controlados pelo captam.

A maior parte das amostras analisadas não continha nas embalagens a origem das sementes. Em apenas 13,8 \% das amostras constavam que as sementes eram importadas; dessas, apenas uma marca comercial indicava o país de procedência. Em algumas das sementes importadas foram detectados Acremonium sp., A. alternata, Aspergillus spp., Cladosporium spp., C. gloeosporioides, Epicoccum 
TABELA 1 - Relação e freqüência relativa ${ }^{a}$ (\%) de fungos detectados em amostras de cinco marcas comerciais de sementes da família Lamiaceae, coletadas no período de 2003 a 2006

\begin{tabular}{|c|c|c|c|c|c|c|c|c|c|c|c|c|}
\hline Fungos & 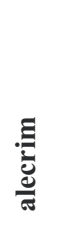 & 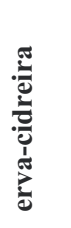 & 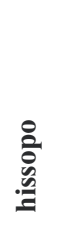 & 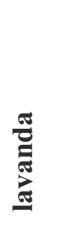 & 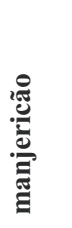 & 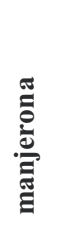 & 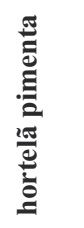 & 营 & 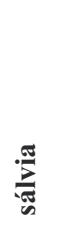 & & 总 & $\stackrel{2}{\frac{\pi}{3}}$ \\
\hline Acremoniella sp. & 0 & 0 & 0 & 0 & 3,6 & 0 & 0 & 0 & 0 & 0 & 0 & 0,8 \\
\hline Acremonium sp. & 0 & 0 & 14,3 & 0 & 0 & 0 & 0 & 0 & 0 & 0 & 0 & 0,8 \\
\hline Alternaria alternata & 50,0 & 15,4 & 14,3 & 0 & 32,1 & 14,3 & 33,3 & 0 & 30,0 & 22,2 & 10,0 & 22,2 \\
\hline Aristatoma oeconomicum & 0 & 0 & 14,3 & 0 & 0 & 0 & 0 & 0 & 0 & 0 & 0 & 0,8 \\
\hline Aspergillus spp. & 75,0 & 0 & 42,9 & 20,0 & 17,9 & 0 & 22,2 & 0 & 30,0 & 22,2 & 0 & 18,8 \\
\hline Bipolaris sorokiniana & 0 & 0 & 0 & 0 & 3,6 & 0 & 0 & 0 & 0 & 0 & 10,0 & 1,7 \\
\hline Chaetomium sp. & 0 & 15,4 & 0 & 0 & 0 & 0 & 0 & 0 & 0 & 0 & 0 & 1,8 \\
\hline Cladosporium spp. & 25,0 & 23,1 & 28,6 & 0 & 42,9 & 14,3 & 22,2 & 27,3 & 40,0 & 22,2 & 40,0 & 29,9 \\
\hline Colletotrichum sp. & 0 & 0 & 0 & 0 & 7,1 & 0 & 0 & 0 & 0 & 0 & 0 & 1,7 \\
\hline C. gloeosporioides & 0 & 0 & 14,3 & 0 & 3,6 & 0 & 11,1 & 0 & 0 & 0 & 0 & 2,6 \\
\hline Curvularia eragrotides & 0 & 0 & 0 & 0 & 0 & 0 & 0 & 0 & 0 & 11,1 & 0 & 0,8 \\
\hline Curvularia inaequalis & 0 & 0 & 0 & 0 & 3,6 & 0 & 0 & 0 & 0 & 0 & 0 & 0,8 \\
\hline Curvularia lunata & 0 & 0 & 0 & 0 & 25,0 & 0 & 0 & 0 & 10,0 & 0 & 0 & 6,0 \\
\hline Curvularia spp. & 0 & 0 & 0 & 0 & 7,1 & 0 & 0 & 0 & 0 & 0 & 0 & 1,7 \\
\hline Doratomyces stemonitis & 0 & 0 & 0 & 0 & 3,6 & 0 & 0 & 0 & 0 & 0 & 0 & 0,8 \\
\hline Epicoccum purpurascens & 37,5 & 15,4 & 28,6 & 0 & 25,0 & 28,6 & 0 & 9,1 & 10,0 & 22,2 & 20,0 & 18,8 \\
\hline Exserohilum sp. & 0 & 0 & 0 & 0 & 7,1 & 0 & 0 & 0 & 0 & 0 & 0 & 1,7 \\
\hline Fusarium equiseti & 0 & 0 & 0 & 0 & 3,6 & 0 & 0 & 0 & 0 & 0 & 0 & 0,8 \\
\hline Fusarium moniliforme & 0 & 0 & 0 & 0 & 0 & 14,3 & 0 & 0 & 0 & 11,1 & 0 & 1,7 \\
\hline Fusarium oxysporum & 0 & 0 & 0 & 0 & 3,6 & 0 & 0 & 0 & 0 & 0 & 0 & 0,8 \\
\hline Fusarium semitectum & 0 & 0 & 0 & 0 & 0 & 0 & 0 & 9,1 & 0 & 0 & 0 & 0,9 \\
\hline Fusarium solani & 0 & 0 & 0 & 0 & 10,7 & 0 & 0 & 9,1 & 0 & 0 & 0 & 2,5 \\
\hline Fusarium spp. & 0 & 7,7 & 0 & 0 & 7,1 & 0 & 0 & 0 & 0 & 11,1 & 0 & 4,3 \\
\hline Fusicoccum sp. & 0 & 0 & 14,3 & 0 & 0 & 0 & 0 & 0 & 0 & 0 & 0 & 0,8 \\
\hline Mucor sp. & 12,5 & 23,1 & 14,3 & 0 & 0 & 0 & 11,1 & 9,1 & 20,0 & 22,2 & 10,0 & 10,3 \\
\hline Penicillium spp. & 75,0 & 0 & 57,1 & 0 & 7,1 & 14,3 & 0 & 9,1 & 40,0 & 0 & 10,0 & 16,2 \\
\hline Pestalotiopsis sp. & 25,0 & 7,7 & 0 & 0 & 0 & 0 & 0 & 0 & 10,0 & 0 & 0 & 3,4 \\
\hline Phoma spp. & 12,5 & 0 & 0 & 0 & 14,3 & 0 & 0 & 0 & 10,0 & 0 & 10,0 & 6,0 \\
\hline Pithomyces chartarum & 0 & 0 & 0 & 0 & 7,1 & 0 & 0 & 0 & 0 & 0 & 0 & 1,7 \\
\hline Rhizopus sp. & 62,5 & 15,4 & 71,4 & 40,0 & 46,4 & 14,3 & 0 & 27,3 & 60,0 & 33,3 & 10,0 & 35,0 \\
\hline Stemphylium botryosum & 0 & 0 & 14,3 & 0 & 0 & 0 & 0 & 0 & 0 & 0 & 0 & 0,8 \\
\hline Trichoderma sp. & 25,0 & 7,7 & 28,6 & 0 & 10,7 & 14,3 & 0 & 0 & 10,0 & 11,1 & 30,0 & 12,0 \\
\hline Ulocladium botrytis & 12,5 & 0 & 0 & 0 & 0 & 0 & 0 & 0 & 0 & 0 & 0 & 0,9 \\
\hline
\end{tabular}

${ }^{\mathrm{a}} \mathrm{Fr}=(\mathrm{n} / \mathrm{N}) \times 100$, onde $\mathrm{n}=$ número de amostras com fungo e $\mathrm{N}=$ número de amostras por espécie de semente.

${ }^{\mathrm{b}}$ a freqüência total refere a presença do fungo nas 117 amostras avaliadas.

purpurascens, F. solani, Fusicoccum sp. e Penicillium spp. Sementes com baixa qualidade sanitária podem favorecer a introdução de patógenos de importância quarentenária e acarretar prejuízos para a agricultura nacional. A presença de fungos nas sementes, a falta de controle desses fungos e a pouca eficiência do fungicida empregado por algumas empresas sementeiras, constatado nas amostras de sementes avaliadas, demonstram a necessidade do estabelecimento de padrões para a comercialização de sementes no país.

Tropical Plant Pathology 33 (1) January - February 2008 
Ocorrência de fungos em sementes de plantas medicinais, aromaticas...

TABELA 2 - Amostras comerciais de sementes da família Lamiaceae, coletadas no município de São Paulo, no período de 2003 a 2006

\begin{tabular}{|c|c|c|c|}
\hline & \multicolumn{3}{|c|}{ porcentagem } \\
\hline & sementes com fungos & sementes sem fungos & total \\
\hline sementes com fungicida ${ }^{a}$ & $29,9(35)^{b}$ & $17,9(21)$ & $47,9(56)$ \\
\hline sementes não tratadas & $41,9(49)$ & $10,3(12)$ & $52,1(61)$ \\
\hline total & $71,8(84)$ & $28,2(33)$ & \\
\hline
\end{tabular}

\section{REFERÊNCIAS BIBLIOGRÁFICAS}

Aziz NH, Youssef YA, El Fouly MZ, Moussa LA (1998) Contamination of some common medicinal plant samples and spices by fungi and their mycotoxins. Botanical Bulletin of Academia Sinica 39:279-285.

Barnett HL, Hunter BB (1972) Illustrated genera of imperfect fungi. 3th. Ed. Minneapolis. Burgess Publishing Company.

Booth C (1971) The Genus Fusarium. Kew, England. CAB International.

Correa Júnior C, Ming LC, Scheffer MC (1994) Cultivo de Plantas Medicinais, Condimentares e Aromáticas. $2^{\text {a }}$. Ed. Jaboticabal SP.

Ellis MB (1971) Dematiaceous hyphomycetes. Kew, England. CAB International.

Gaetan SA, Gally ME (1993) Anthracnose of peppermint (Mentha piperita L.) caused by Colletotrichum gloeosporioides (Penz.) Sacc. Boletin-de-Sanidad-Vegetal 19: 673-676.

Garbagnoli C, Gaetan SA (1994) Wilting of marjoram (Origanum vulgare L.) caused by species of the genus Fusarium in Argentina. Fitopatologia 29:150-155.

Garibaldi A, Gullino ML, Minuto G (1997) Diseases of basil and their management. Plant Disease 81:124-132.

Kruppa PC, Russomanno OMR (2001) Fungos associados à sementes de manjericão (Ocimum basilicum). Arquivos do Instituto Biológico 68(Supl.):57.

Machowicz-Stefaniak Z, Zalewska E, Zimowska B (2004) Fungi colonizing above-ground parts of lemon balm (Melissa officinalis L.) and thyme (Thymus vulgaris L.) cultivated in the Lublin Region. Folia Universitalis Agriculturae Stelinemsis 95:229-232.

Machowicz-Stefaniak Z, Zalewska E, Zimowska B (2002) Fungi colonizing various organs of lemon balm (Melissa officinalis L.) cultivated in South-East Poland. Plant Protection Science 38 (Special 2):347-350.

Malone JP, Muskett AE (1964) Seed-borne fungi. Description of 77 fungus species. Proceedings of the International Seed Testing Association, 29:179-384.
Mendes MAS, Silva VL, Dianese JC, Ferreira MASV, Santos CEN, Gomes Neto E, Urben AF, Castro C (1998) Fungos em Plantas no Brasil. Brasília DF. Embrapa.

Mendes MAS, Vieira RF, Oliveira AS, Santos JKP (2003) Murcha de Fusarium em manjericão no DF. Fitopatologia Brasileira 28 (Supl.):219-220.

Neergaard P (1979) Seed Pathology. Vol. 1. London. The MacMillan Press.

Perello A, Dal Bello GM (1995) Foliar necrosis caused by Alternaria alternata on rosemary and Colletotrichum spp. on lavender, sage and marjoran. Investigacion Agraria, Produccion y Proteccion Vegetales 10:275-281.

Richardson MJ, Noble M (1968) An annotated list of seed-borne diseases. 2th. Ed. London. Commonwealth Mycological Institute.

Russomanno OMR, Kruppa PC, Martins A, Figueiredo MB (2004a) Murcha de Fusarium oxysporum em plantas de alecrim (Rosmarinus officinalis) e manjericão anão (Ocimum minimum). Summa Phytopathologica 30:98. (Resumo)

Russomanno OMR, Kruppa PC, Valesan AMC, Melinski AR, Isikawa PM (2004b) Ocorrência de fungos em chás industrializados. Summa Phytopathologica 30:98. (Resumo)

Singh K, Frisvad JC, Thrane U, Mathur SB (1991) An Illustrated Manual on Identification of some Seed-borne Aspergilli, Fusaria, Penicillia and their Mycotoxins. Jordbrugsforlaget, Frederiksberg, Denmark.

Souza VC (2005) Botânica Sistemática: Guia Ilustrado para Identificação das Famílias de Angiospermas da Flora Brasileira. Nova Odessa SP. Instituto Plantarum.

Surviliene E, Dambrauskiene E (2006) Effect of different active ingredients of fungicides on Alternaria spp. growth in vitro. Agronomy Research 4 (Special Issue):403-406.

Urben AF, Mattos JKA, Mendes MAS (1987) Fungos associados a manchas de folhas em plantas de uso medicinal, no Distrito Federal. Fitopatologia Brasileira 12:390-394. 